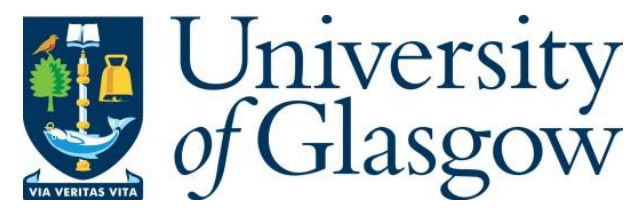

Lay, H. S., Seetohul, V., Cox, B., Demore, C. E.M., and Cochran, S. (2014) Design and Simulation of a High-Frequency Ring-Shaped Linear Array for Capsule Ultrasound Endoscopy. In: 2014 IEEE International Ultrasonics Symposium (IUS), Chicago, IL, USA, 3-6 Sep 2014, pp. 683-686. (doi:10.1109/ULTSYM.2014.0168)

There may be differences between this version and the published version. You are advised to consult the publisher's version if you wish to cite from it.

http://eprints.gla.ac.uk/129625/

Deposited on: 05 October 2016

Enlighten - Research publications by members of the University of Glasgow http://eprints.gla.ac.uk 


\title{
Design and Simulation of a High-Frequency Ring-Shaped Linear Array for Capsule Ultrasound Endoscopy
}

\author{
Holly S. Lay, Vipin Seetohul, Ben Cox, Christine E. M. Démoré, Sandy Cochran \\ IMSaT, Medical Research Institute \\ School of Medicine, University of Dundee \\ Dundee, UK \\ h.lay@dundee.ac.uk
}

\begin{abstract}
Current research into endoscopy and colonoscopy has significantly advanced visualization of the gastrointestinal tract (GIT). The Sonopill project seeks to combine the imaging capabilities of endoscopic ultrasound with the full GIT transit of capsule endoscopy through the development of a capsule capable of ultrasonic imaging of the GIT, focusing on the small intestine. However, due to the small volume of the proposed capsule and the need to transmit received data wirelessly, the Sonopill system is limited both in data bandwidth and power. This paper presents a MATLAB-based simulation to allow testing of transducer topologies and imaging methodologies to achieve optimum results within the physical limitations of the system. To allow rapid evaluation of possible transducer configurations and circuit elements, a hybrid MATLAB simulation was created, incorporating both KLM circuit elements for analog analysis and digitizing and beamforming elements to render a final grey-scale image for imaging quality analysis. This was used in conjunction with a theoretical acoustic propagation model to image ideal point scatterers. The proposed transducers consist of a single, unfocused transmit ring of radius $5 \mathrm{~mm}$ separated into eight segments for impedance control, and a 512-element receive linear array curved into a matching ring. Because of the high element count and pad limitations on the intended electronics, the design requires the use of 32 integrated 16:1 multiplexers which will be bonded directly to the connecting flex circuit before the ASIC. Simulating the loading effects of these multiplexers as well as the proposed transducer configuration was critical to the analysis of the design. The MATLAB model was used to simulate a standard pulser transmitting over a $2.5 \mathrm{~m}$ cable to a $0.25 \mathrm{~mm} \times 8 \mathrm{~mm} \times 85$ $\mu \mathrm{m}$ PMN-PT piezocrystal transmit transducer with a centre frequency of $25 \mathrm{MHz}$. B-scan images were then modelled for three imaging phantoms, one containing three point target resolution phantoms, a resolution phantom containing two virtual walls, and a tissue mimicking phantom containing particles with two levels of reflectivity to represent a three layer gut phantom with a highreflectivity front surface.
\end{abstract}

Keywords - endoscopic ultrasound; high resolution ultrasound; system modelling; KLM; capsule endoscopy.

The work presented in this paper was completed under the SONOPILL grant funded by the UK EPSRC.

\section{INTRODUCTION}

Endoscopic imaging to assist the visualization and diagnosis of diseases of the gastrointestinal tract (GIT) has been in existence for over 200 years, with subsequent development to incorporate high-quality optics, fibre-optic light sources and high-definition cameras. In 2001, a wireless optical capsule endoscope capable of passing entirely through the GIT and transmitting the images in real-time was approved for human use, giving clinicians the first access to non-invasive imaging of the internal structure of the GIT. Current research is investigating the ability to expand the diagnostic capabilities of these endoscopic capsules in many ways, but particularly with high-resolution ultrasound. While the use of ultrasonic imaging is common in conjunction with standard endoscopic imaging, as yet no form of ultrasonic endoscopic pill exists on the market [1].

When imaging the wall of the GIT, it is clinically important to be able to distinguish the various layers and their characteristics for diagnostic purposes [2]. As the gut wall is relatively thin, minimizing depth-based signal attenuation, this makes capsule endoscopic ultrasound an ideal application for high-resolution ultrasonic imaging.

Due to the complexity of manufacturing high-frequency ultrasonic transducers for miniaturized devices, early modelling is critical to produce an optimized device design that has the best possibility of producing high quality images. While finite element analysis does well at replicating the full material characteristics of a design, it suffers from long simulation times and high computational expense. Previous work has shown good agreement between simpler, one-dimensional models such as KLM and fabricated transducers [3 - 5], allowing a much larger set of designs to be tested in a reasonable timeframe. 


\section{ENDOSCOPIC ULTRASOUND ARRAY}

\section{A. Circumferential Array}

When considering an application-specific ultrasound array, it is necessary to take into account the geometrical requirements of the final probe shape when deciding on an overall array design. In the case of a pill-based imaging system, the final array should conform to the outside surface of the capsule itself. For this particular system, the decision was made to design a side-looking circumferential array to best image into the thickness of the gut wall without requiring physical rotation of the probe or the array. To achieve a compromise between sidelobe levels and electronic complexity, a 512-element array was selected. With a capsule diameter of $10 \mathrm{~mm}$, this results in a pitch of $61.4 \mu \mathrm{m}$. While this number of elements is not unknown in the commercial market, it does contribute additional connectivity concerns due to limitations in electronics dimensions within the pill cavity.

\section{B. Synthetic Aperture and De-Focused Transmit}

Due to the size restrictions of the electronics and interconnections which can be fit within the endoscopic capsule, the desire was for a system which would require only one active transmit and receive channel on each pulse. While this adds additional interconnection and multiplexing complexity, the active circuit power load and footprint is significantly reduced over a fully sampled array. However, this can have a significant impact on the scan rate of the system because of the need for multiple passes to completely sample the aperture.

To understand the scan rate limitations of the system, the expected maximum movement rate of the capsule was assessed. In the human GIT, the average rate of motion through the system is approximately $0.17 \mathrm{~mm} / \mathrm{sec}$ [6]. However, as this motion can be highly pulsatile, especially in abnormal systems, a much higher rate of $2-5 \mathrm{~mm} / \mathrm{s}$ was assumed for the purposes of system design. Assuming a pulse-echo time of $50 \mu$ s and 512 scan lines, a full B-scan image would take $25.6 \mathrm{~ms}$ to complete. In this time, even at the faster rate of $5 \mathrm{~mm} / \mathrm{s}$, the capsule will have moved less than $130 \mu \mathrm{m}$, minimizing distortion at the B-scan zero angle and allowing a maximum frame rate of 39 frames/sec. However, this frame rate would be reduced by a factor the size of the aperture if full synthetic aperture imaging were used. Instead, a compromise is proposed in which a large, de-focused transmit element is used in conjunction with individually sampled receive elements, maintaining the frame rate of traditional imaging while attaining the reduction in electronic complexity offered by a synthetic aperture.

\section{SYSTEM MODELLING}

\section{A. KLM Model}

To replicate both the electrical and acoustic properties of the piezoelectric transducer, as well as its accompanying electrical circuitry, the KLM [7] approach was used simulate the piezoelectric and electronic components of the system in MATLAB (The Mathworks, Cambridge, UK). In particular, care was taken to model the parasitic effects of the multiplexers [8] used on the receive end to implement the synthetic aperture. The final model, seen in Fig. 1, also incorporates the effects of

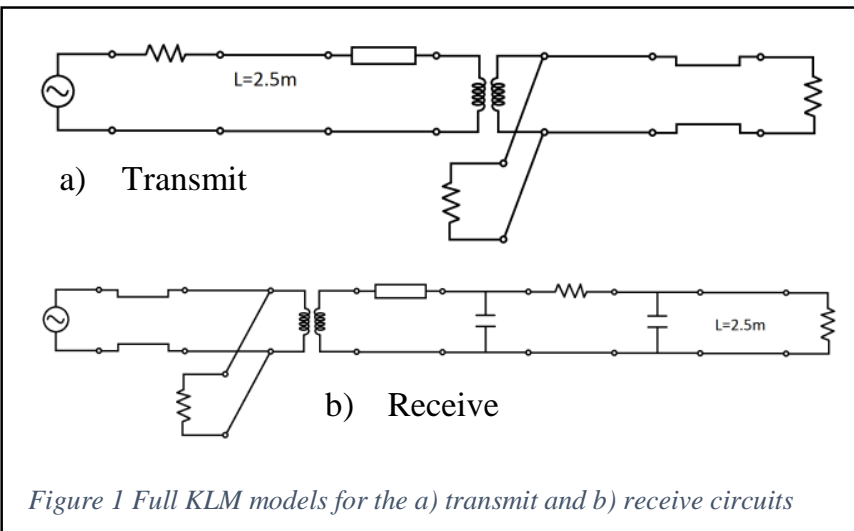

a $2.5 \mathrm{~m}$ coaxial tether which will feature in the initial prototypes to facilitate power and data transfer.

\section{B. Beamformer}

To simulate the digital components of the proposed system, a MATLAB-based beamformer was created to interface with the analogue KLM model and allow virtual phantoms to be imaged. This beamformer also includes the effects of a 12 bit, $100 \mathrm{MS} / \mathrm{s}$ analogue to digital converter (ADC). Modelling this digitization effect is particularly important, as it can affect the accuracy of the beamforming delays.

The beamformer was designed to allow the aperture size, windowing, directivity cut-off and other imaging parameters to be adjusted to assess the imaging abilities of the system. Imaging phantoms were then created by defining point reflectors with individual reflection coefficients and calculating the reflected input to the receive KLM circuit by superposition.

\section{RESULTS}

\section{A. Transducer Impedance and Time-Domain Pulse-Echo}

The KLM model described previously was stimulated using a single cycle transmit sinusoid centred at $25 \mathrm{MHz}$. The input impedance of individual transmit and receive elements was then calculated across a frequency sweep from baseband to $50 \mathrm{MHz}$. These calculations were performed only on the piezo-electric components of the transducers and neglect the influence of the electrical components, showing the unloaded resonance and impedance of the transducers. PMN-PT was selected as the material of choice for simulation, and the geometric mean of the acoustic impedances was used to calculate the desired matching layer impedance. The thicknesses of both the piezoelectric and matching layers were then tuned to obtain an acceptable resonance curve. After tuning, a PMN-PT thickness of $85 \mu \mathrm{m}$ and a matching layer $26 \mu \mathrm{m}$ thick with an acoustic impedance of 4.08 MRayl produced the simulated impedance curves in Figs. $2 \mathrm{a}$ and $2 \mathrm{~b}$.

Once an acceptable pulse-echo response had been simulated, KLM code simulating a $2.5 \mathrm{~m}$ coaxial transmission line was added to both transmission and reception paths, as well as multiplexer modelling on the receiver. For the purposes of modelling, the ADG706 (Analog Devices, Norwood, MA, USA) was selected as a suitable 16:1 multiplexer and the 


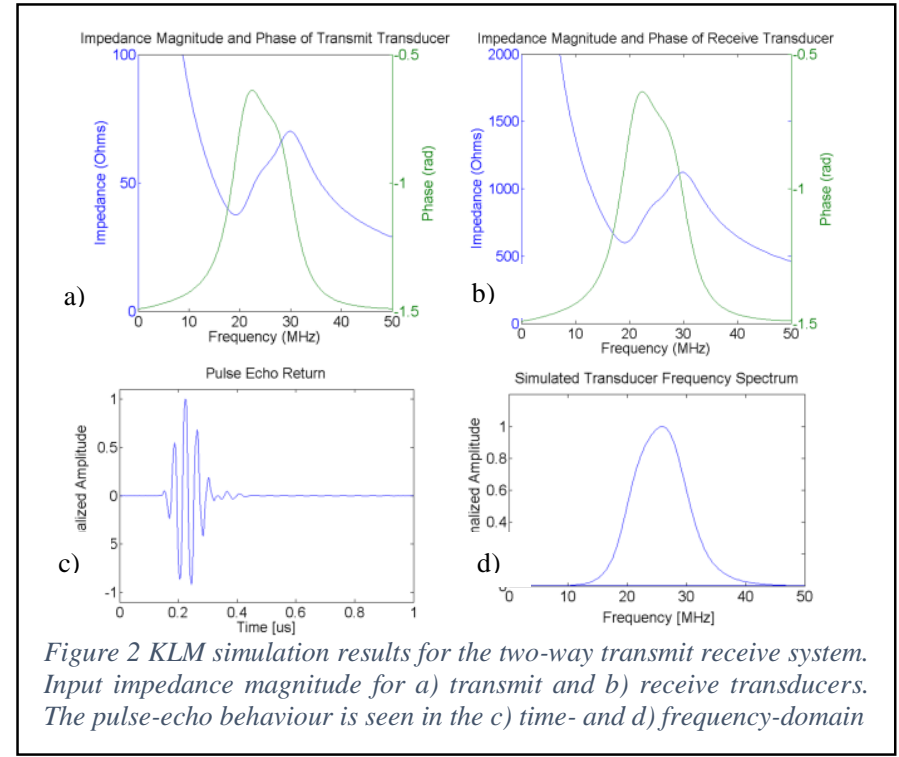

specified on resistance and parasitic capacitance modelled. The two-way pulse-echo response was then calculated from an ideal point-scatterer target and the pulse bandwidth and time-domain pulse were plotted, as shown in Figs. $2 \mathrm{c}$ and $2 \mathrm{~d}$. A bandwidth of $44.0 \%$ was calculated for the two-way system with a centre frequency of $24.9 \mathrm{MHz}$.

\section{B. Model Verification}

To verify that the KLM modelling code developed was an accurate model of the expected system behavior, a known, single-element transducer was modelled using the same methodology, and the resultant resonance curves compared. As shown in Fig. 3, the model has a peak at $44.0 \mathrm{MHz}$ at an impedance of $18.4 \Omega$, while the experimental data shows a peak at $43.1 \mathrm{MHz}$ at an impedance of $18.2 \Omega$, in good correspondence.

\section{Imaging Resolution}

To assess the imaging resolution of the system, as well as the effects of the defocused transmit pulse, a virtual phantom was generated with three ideal reflectors located at $(1.5 \mathrm{~cm}, 0)$, $(2 \mathrm{~cm}, \pi / 10)$ and $(2.5 \mathrm{~cm},-\pi / 10)$, corresponding to the $\mathrm{f} / 2, \mathrm{f} / 3$ and $f / 4$ points for the receive aperture. A linear array scan was then performed with an aperture of 81 elements and a focal point of $1.5 \mathrm{~cm}$, resulting in an effective f-number, $\mathrm{f} \#=2$ because of the curvature of the array. The resulting grey-scale B-scan was displayed with a dynamic range of $60 \mathrm{~dB}$ and standard log-compression, Fig. 4. The three point targets had FWHM of $1.26 \times 10^{-2}, 1.47 \times 10^{-2}$ and $1.74 \times 10^{-2} \mathrm{rad}$ respectively and the simulation was completed in $101 \mathrm{sec}$.

As the intended application of the array will target tissues featuring well defined wall-type structures with relatively thin layers, a second virtual phantom was created to simulate two circular walls around the array. The two walls were each defined to consist of 360 ideal reflectors arranged around the array at radial distances of 1.5 and $1.6 \mathrm{~cm}$ from the centre of the array. A sinusoidal aberration was added to the wall definition to mimic the irregularity of normal tissue layers. The resulting Bscan was produced in $22.4 \mathrm{~min}$ and shows good distinction between the two walls, Fig. 5 .

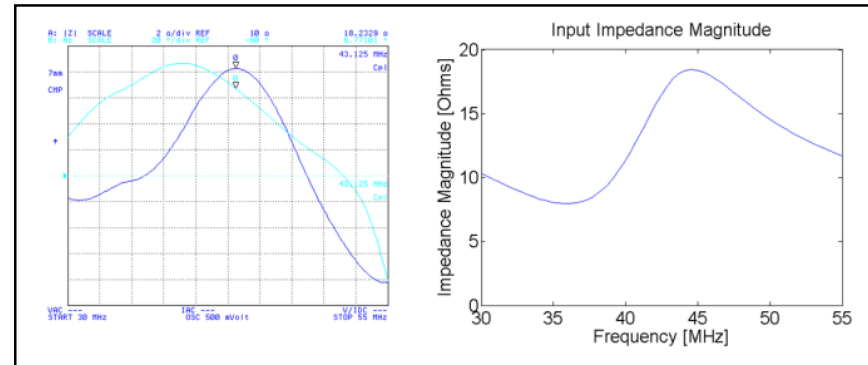

Figure 3 Comparison of KLM resonance calculations versus known transducer.

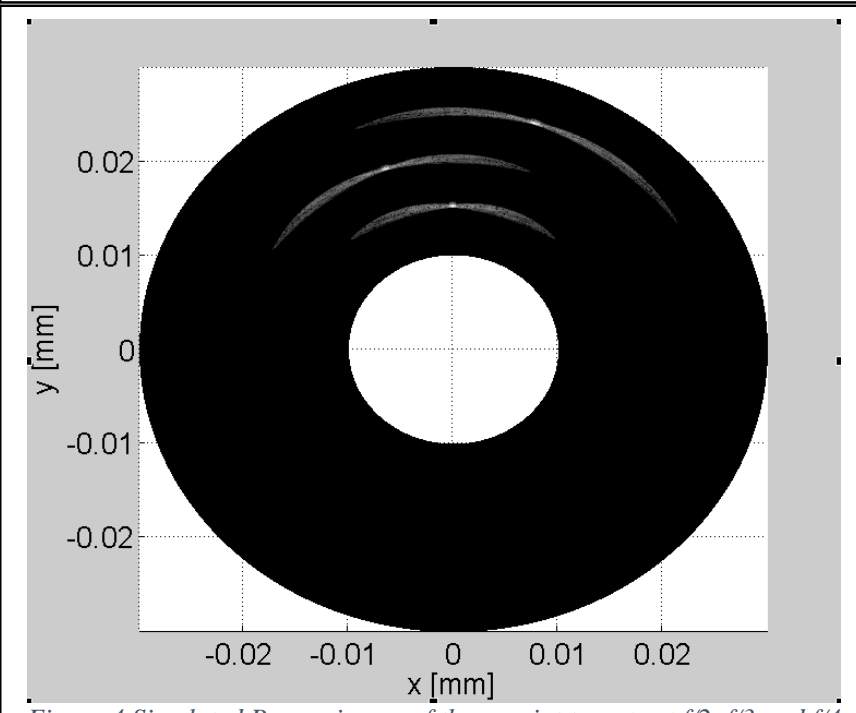

Figure 4 Simulated B-scan image of three point targets at $f / 2, f / 3$ and $f / 4$ displayed with $60 \mathrm{~dB}$ of dynamic range

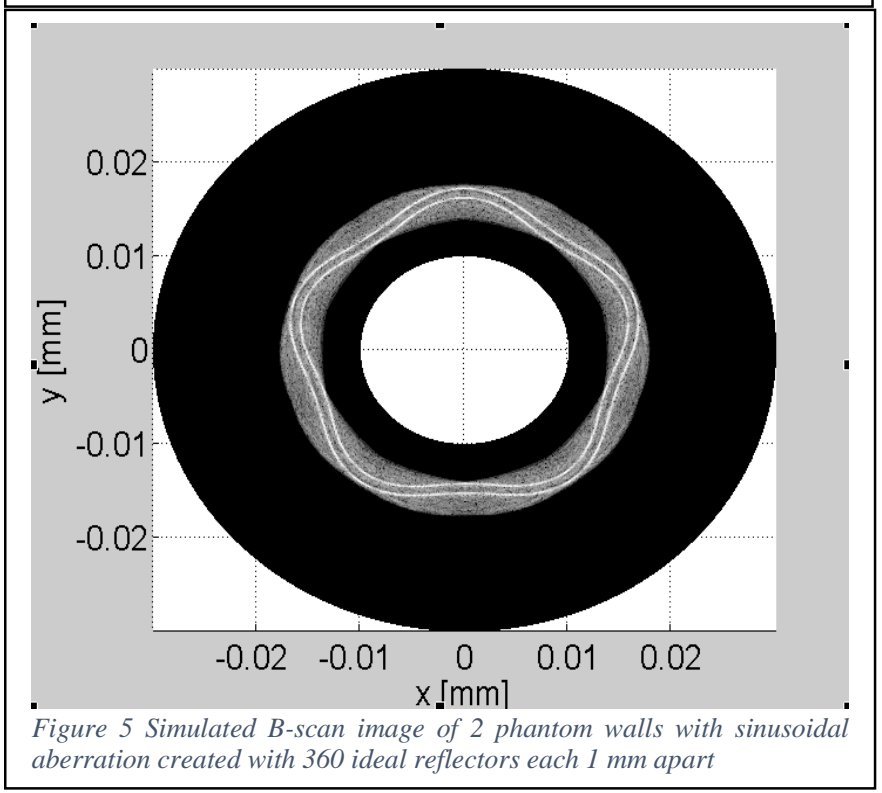

\section{Virtual Tissue Images}

Once the imaging resolution had been established, a virtual tissue phantom was created to assess the tissue imaging abilities of the system. 10,000 pseudo-randomly generated reflectors were placed in a cylindrical area from $1.5 \mathrm{~cm}$ to $3.0 \mathrm{~cm}$ in radius and $1 \mathrm{~mm}$ in thickness, centred around the 


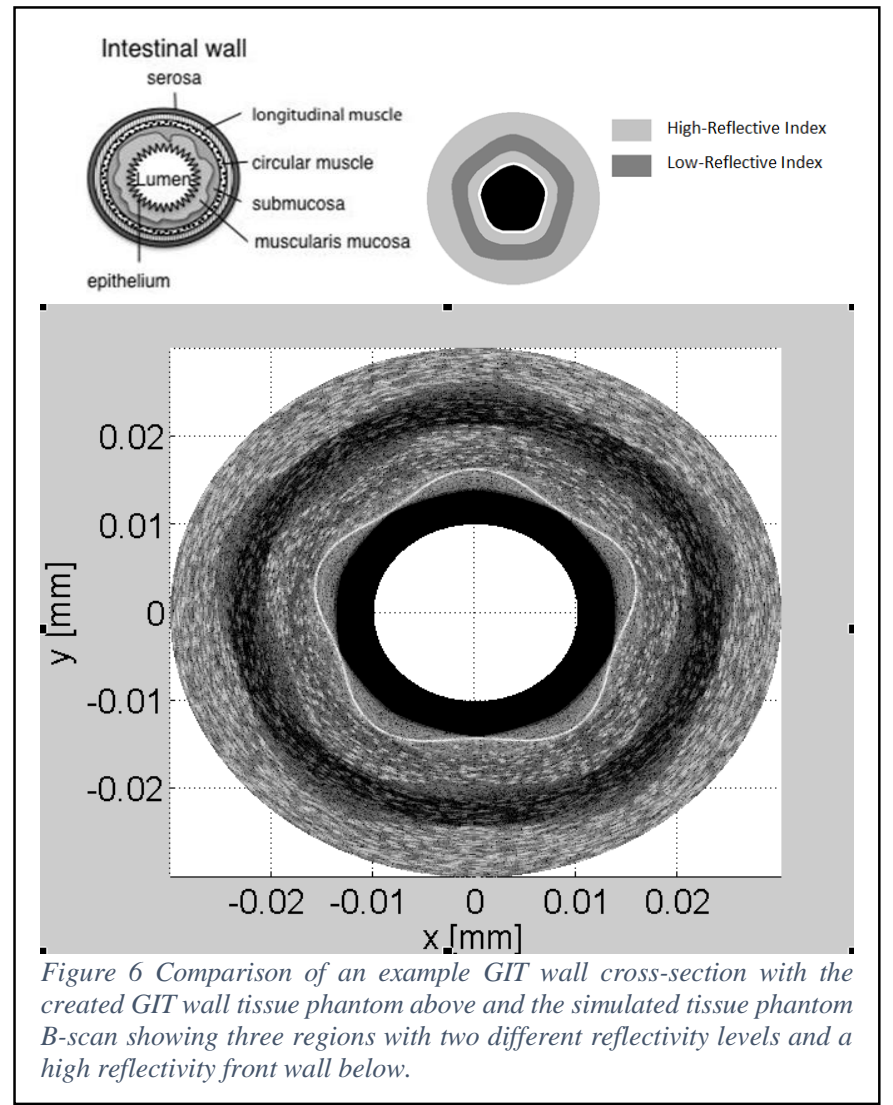

array and in the same plane. Three regions and a front face were then created, with the front face having a reflectivity of 1.0, the central region having a reflectivity of 0.1 and the front and back regions having reflectivities of 0.01 . This tissue phantom was then imaged using the full system model in 222 minutes, with the resulting B-scan shown in Fig. 6.

\section{DISCUSSION AND CONCLUSIONS}

By integrating one-dimensional KLM modelling with a full digital beamformer implemented in MATLAB, a virtual system was created which is capable of assessing the electronic and acoustic performance of the proposed endoscopic ultrasound capsule system. The $<2 \mathrm{~min}$. calculation time for resolution phantoms allows multiple design configurations to be tested and compared in a reasonable amount of time.

The phantom simulations show that, while the expected broadening of the point-target side-lobes can be seen because of the use of a defocused transmit pulse, the twin walls in the bright phantom and the differentiation in the tissue phantom are still distinguished clearly, suggesting that the system will have the imaging ability to allow GIT disease diagnosis.

\section{ACKNOWLEDGMENT}

The authors would like to thank Srikanta Sharma for supplying transducer data for the purposes of verification.

\section{REFERENCES}

[1] G. Ciuti, A. Menciassi, P. Dario, "Capsule endoscopy: from current achievements to open challenges," IEEE Rev. Biomed. Eng., vol. 4, pp. 59-72, 2011.

[2] S. Ødegaard, L. B. Nesje, O. D. Lærum, M. B. Kimmey, "High-frequency ultrasonographic imaging of the gastrointestinal wall”, Expert Rev. Med. Devices 9(3), pp. 263-273, 2012.

[3] F. S. Foster, L. K. Ryan, D. H. Turnbull, "Characterization of lead zirconate titanate ceramics for use in miniature high-frequency (20-80 MHz) transducers", IEEE Trans. Ultra. Ferro and Freq. Cont., Vol. 38, No. 5, pp. 446-453, 1991.

[4] M. J. Zipparo, K. K. Shung, T. R. Shrout, "Piezoceramics for high frequency (20 to $100 \mathrm{MHz}$ ) single-element imaging transducer", IEEE Trans. Ultra. Ferro and Freq. Cont., Vol. 44, No. 5, pp.1038-1048, 1997.

[5] H. S. Lay, E. A. Simpson, G. Griffin, G. R. Lockwood, "High-frequency annular array fabrication using a flex circuit matching layer." Ultrasonic Imaging Jul; 34(3):196-204, 2012.

[6] J. E. Hall, Guyton and Hall Textbook of Medical Physiology, $12^{\text {th }}$ ed., Elsevier, pg.769, 2011.

[7] R. Krimholtz, D. A. Leedom, G. L. Matthaei, "New equivalent circuits for elementary piezoelectric transducers", Electronics Letters, $27^{\text {th }}$ May, pp. 398-399, 1970

[8] L. J. Busse, C. G. Oakley, M. J. Fife, J. V. Ranalletta, R. D. Morgan, D. R. Dietz, "The acoustic and thermal effects of using multiplexers in small invasive probes”, IEEE Ultrasonics Symposium, pp. 1721-1724, 1997. 